\title{
Détection du biotope larvaire de Leptoconops (Holoconops) kerteszi Kieffer, 1908 (Diptera-Ceratopogonidae) dans le "Midi" méditerranéen
}

\author{
par Jean-A. RIOUX et Suzanne DESCOUS
}

Répandus dans le monde entier, les Cératopogonides du genre Leptoconops Skuse, 1890 , constituent par place (zones arides, régions lagunaires) un véritable fléau. Leur grand nombre et leur petite taille rendent toute défense individuelle impossible. Leur transport à longue distance par les vents et par les animaux (H. Foley et A. PicoutLaforest, 1923) explique les difficultés auxquelles se heurtent toutes les tentatives classiques de lutte insecticide.

Leur intérêt vétérinaire et médical est également grand: certains [Leptoconops (Holoconops) mediterraneus Kieffer, 1921] serviraient d'hôtes intermédiaires à la filaire de l'âne (H. Foley et A. Picout-Laforest, 1923). Dans les zones très infestées, ils peuvent être à l'origine d'irritations (S. Mulla, 1958) ou d'adénomégalies (C. Hannoun, D. Beytout, L. Joubert, J. Mouchet et E. Abonnenc, 1964), et il n'est pas exclu que quelques espèces soient responsables de la transmission à l'homme d'affection à virus arbor.

En Camargue et en Languedoc-Roussillon, les Leptoconops sont connus de temps immémoriaux sous le nom d" " arabis », vocable évoquant vraisemblablement les multiples et meurtrières invasions sarrazines. A certaines périodes, leur agressivité atteint une telle intensité qu'elle oblige à interrompre les travaux des champs et vide les plages de leurs baigneurs.

La lutte anti-culicidienne dont les résultats sont assez sensibles sur la bordure littorale du Gard, de l'Hérault, de l'Aude et des Pyrénées-Orientales, n'a aucun effet sur eux.

Aussi bien, dans le but de diminuer leur nuisance par les moyens chimiques ou physiques, était-il important de recenser les principales espèces du « Midi » méditerranéen, d'en étudier la répartition et d'en préciser l'écologie. 
Jusqu'à présent six espèces ont été signalées sur nos côtes :

Leptoconops (Leptoconops) irritans Noe, 1899 (?).

Leptoconops (.) lisbonnei Harant et Galan, $1943\left({ }^{\star}\right)$.

Leptoconops (Holoconops) mediterraneus Kieffer, 1921 (†).

Leptoconops (Microconops) vexans Kieffer, 1921 (†).

Leptoconops (Holoconops) kerteszi Kieffer, 1908 (ㅇ).

Leptoconops (.) inopinatus Huttel et Huttel, 1951 (ठో).

Notons que pour chacune d'entre elles, seuls le mâle ou la femelle ont été décrits ou signalés. Aussi avec quelque raison, d'abord H. Harant et G. Galan (1943), ensuite W. Huttel et N. Huttel (1951) se demandent à propos de descriptions nouvelles, s'ils ne se trouvent pas en présence de mâles d'une espèce déjà connue par ses femelles.

Nous présentons ici les premiers résultats de nos recherches sur la plus vulnérante d'entre elles, observée à l'état larvaire et adulte, Leptoconops (Holoconops) kerteszi Kieffer, 1908.

\section{RAPPEL MORPHOLOGIQUE}

Leptoconops (Holoconops) kerteszi Kieffer, 1908 appartient à la sous-famille des Leptoconopinae Enderlein, 1936 qui groupe classiquement les Cératopogonides à nervure alaire radio-médiane $(r-m)$ absente. Il s'intègre par ailleurs dans le sous-genre Holoconops Kieffer, 1908 caractérisé chez la femelle par des antennes à 13 articles (au lieu de 14 ou 12 dans les autres sous-genres) et des métatarses antérieurs spinuleux.

\section{Larve.}

Larve d'environ $5 \mathrm{~mm}$ de longueur, extrêmement mobile, nageant activement dans les sables gorgés d'eau. Tête petite, légèrement chitinisée, suivie de treize segments.

\section{Mâle.}

Yeux nus. Espace interoculaire large $(142 \mu), 4$ soies sur le fronto-clypeus. Antennes de 15 articles, III-XV passant progressivement de transversaux à longiformes. XV de beaucoup le plus long $(150 \mu)$ s'élargissant en forme de massue. Palpes de 4 articles. I hyalin, II avec une plaque fortement chitinisée d'où sortent 2 ou 3 fortes soies, III-IV chitinisés, I-II sub-égaux (ensemble $100 \mu$ ), III-IV sub-égaux (ensemble $150 \mu$ ). Aile allongée, petite $(1 \mathrm{~mm})$, hyaline, recouverte de microtriches et bordée d'une frange, excepté sur une certaine longueur à l'extrémité de la costale. Nervures longitudinales très nombreuses mais difficilement visibles. Seule la bifurcation de la cubitale se voit facilement. Abdomen se rétrécissant brusquement vers le $5^{\circ}$ segment. Genitalia caractéristiques (fig. 1). En particulier, $9^{\circ}$ tergite présentant une paire de processus apicaux et médians en forme de pinces. 


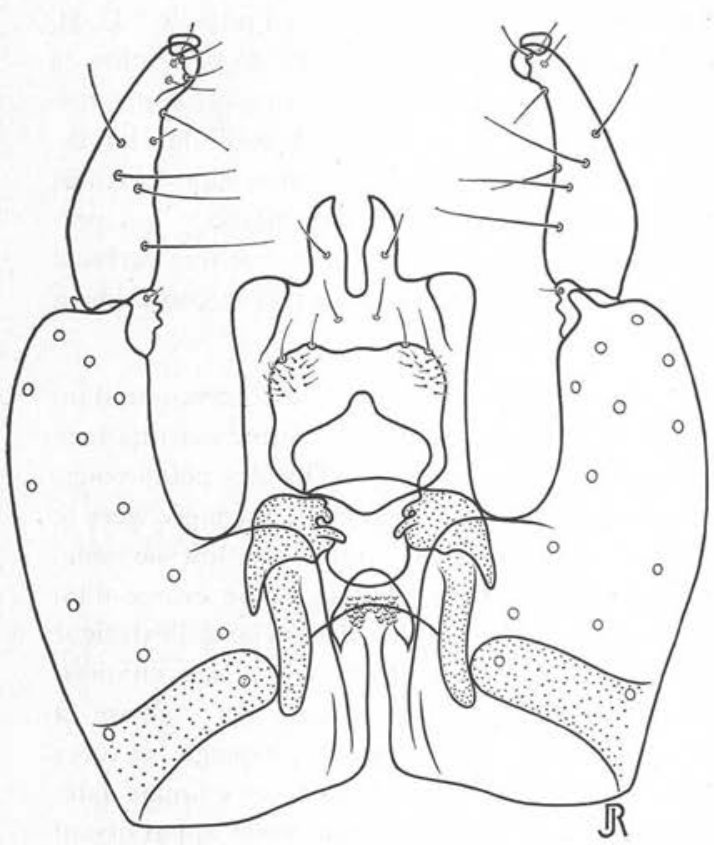

Fig. 1. - Leptoconops (Holoconops) kerteszi Kieffer, 1908: hypopygium
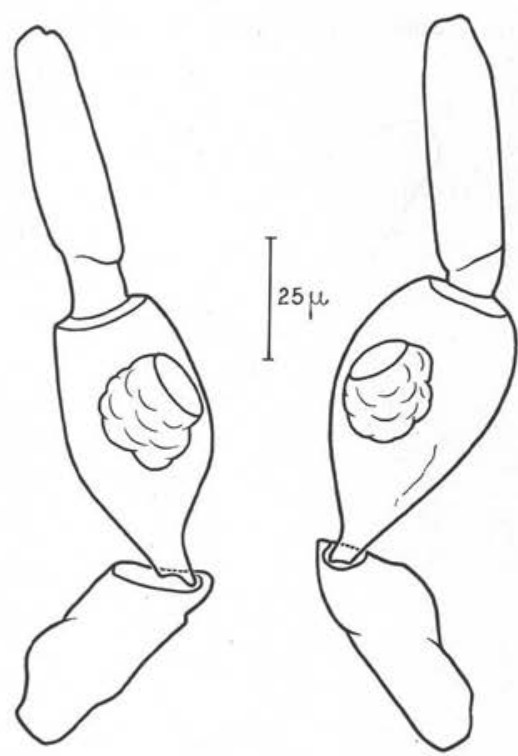

FIG. 2. - Leptoconops (Holoconops) kerteszi Kieffer, 1908 : palpe +

\section{Femelle.}

Yeux nus, espace interoculaire large $(105 \mu)$. Soies fronto-clypéales peu fournies. Antennes (fig. 3) de 13 articles, III-IX allant de nettement transversaux à globuleux, X globuleux, XI-XII un peu allongés, XIII le plus long $(60 \mu)$, se termine en massue. Palpes (fig. 2), 4 articles, I et II peu différenciés, II, avec 3 fortes soies identiques à celles du mâle, III fortement renflé avec une fossette olfactive, IV allongé cylindrique. III-IV sub-égaux (ensemble $105 \mu$ ). Métatarse antérieur avec 2 à 8 spinules noires. Griffes égales et simples. Aile hyaline à microtriches bordée d'une frange. Nervures plus visibles que chez le mâle. Base de la radiale assez souvent de teinte orangée à rouge avec un stigma de même couleur. Abdomen effilé. Deux spermathèques normalement développées ; une spermathèque rudimentaire. Cerques longs $(205 \mu)$ et grêles.

\section{METHODE DE CAPTURE}

Dans un premier temps nous avons utilisé sans succès la méthode d'élevage en aquaterrarium hermétique (H.-F. Carter, A. Ingram et J.-W.-S. Macfie, 1920). Si ce procédé permet d'obtenir en effet de belles récoltes de Culicoides, Bezzia et Dasyhelea (J. Callot et M. Kremer, 1960), il est par contre à peu près inefficace à l'encontre des 
Leptoconops. Par la suite, la lecture des travaux américains (R. E. Fontaine, D. H. Green et L. M. Smith, 1957) nous amenait à mettre sur le compte de cet échec la grande profondeur du gîte [entre 30 et $90 \mathrm{~cm}$ pour Leptoconops (Leptoconops) tor-

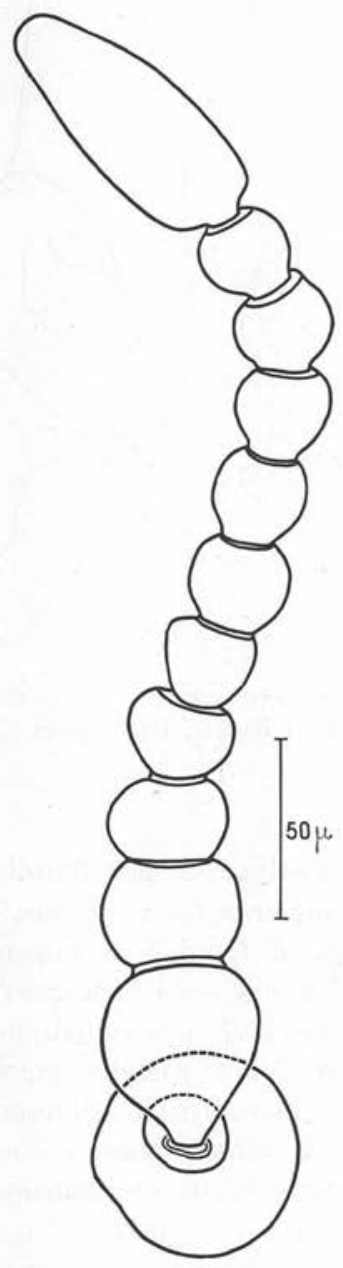

Fig. 3. - Leptoconops (Holoconops) kerteszi Kieffer, 1908: antenne ? rens Townsend, 1893] et la faible densité des larves. Changeant de procédé, nous nous sommes alors orientés vers le piégeage direct sur le terrain, méthode qui permettait de récolter tout diptère naissant sur une certaine surface de sol et ce, quelle que soit la profondeur à laquelle se tenaient les larves.

Le piège, construit à cet effet, est composé d'un cadre en bois de $100 \times 55 \times 20 \mathrm{~cm}$, fermé sur une face par un rectangle de toile noire. Sur l'un des petits côtés, une ouverture de $6 \mathrm{~cm}$ de diamètre est ménagée vers le bord supérieur, c'est-à-dire au contact du toit de toile. Dans cette ouverture, on introduit la partie évasée d'un entonnoir dont l'extrémité effilée débouche à l'extérieur dans un récipient étanche et transparent (sac en plastique, Erlen-Meyer ou tube à essais) de manière à constituer une sorte de nasse (fig. 4). Le piège est alors déposé à plat sur le sol, toile en haut, et colmaté latéralement de telle sorte que tout arthropode apparaissant sur la surface couverte se dirige obligatoirement dans la nasse (phototactisme). Chaque matin la récolte est relevée, étiquetée et mise en alcool.

Grâce à cette méthode, nous avons pu inventorier les différents biotopes d'une localité périodiquement envahie par les "arabis», localité située au S-E du Grau-du-Roi (Gard), en bordure de la mer [Domaine de La Figueirasse (1)]. Dans ce domaine, implanté sur des sables marins relativement fins, se trouvent en effet réunis une grande variété de milieux, depuis les terres cultivées (vignes et aspergeries) jusqu'aux zones incultes (pinèdes à Pinus pinea L., maquis à Juniperus phoenicea $L$., dunes mobiles et fixées, « joncasses », " souillères » à Salicornes, lagunes, plages côtières).

Au cours des mois de mai, juin et juillet 1964, nous avons réparti nos pièges en vue d'explorer les stations suivantes :

(1) A nos amis R. et F. Saumade, qui nous ont aimablement accueillis dans leur domaine de La Figueirasse, nous adressons nos plus vifs remerciements. 
- fossé de $1 \mathrm{~m}$ de profondeur bordé de peupliers entre deux champs d'asperges,

- sable nu entre une dune fixée et un champ d'asperges,

- bas-fond sableux à Phragmites communis Trin. entre deux dunes fixées,

- sable nu entre une dune fixée et une vigne,

- marais à Juncus maritimus Lamk. et Juncus acutus L.,

- plage sableuse en bordure de lagune.

Dans toutes ces stations nous avons piégé un grand nombre de diptères dont plusieurs espèces de Cératopogonides (Culicoides, Dasyhelea, Forcipomyia). Seules les plages de sable bordant les lagunes ont permis d'obtenir les adultes ( $\sigma^{*}$ et $\uparrow$ ) et les lar-

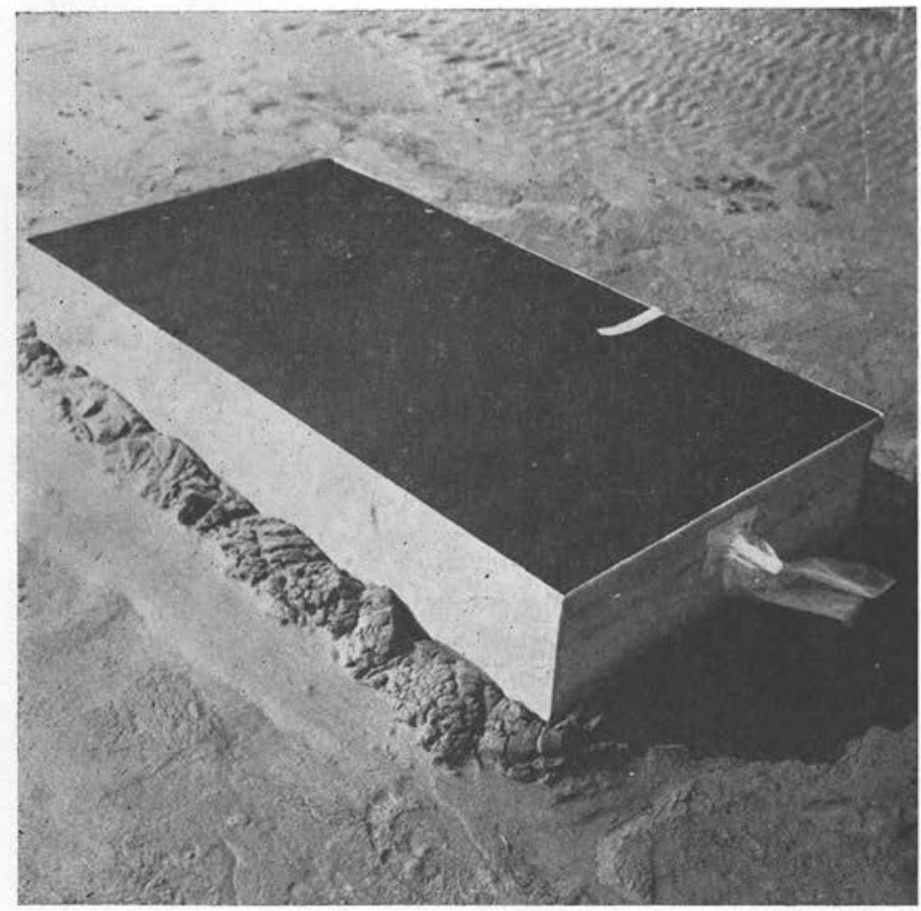

FIG. 4. - Piège à Leptoconops en place sur une grève lagunaire (station $\mathrm{n}^{\circ}$ 7)

ves de Leptoconops. Il s'agissait de Leptoconops (Holoconops) kerteszi Kieffer, 1908. Dans ce biotope, nous avons alors groupé la totalité des pièges de manière à étudier la localisation des larves par rapport à la profondeur de la nappe phréatique et à l'éloignement de l'eau libre (fig. 5). Des prélèvements itératifs de sols et d'eau effectués à différentes profondeurs ont permis de réaliser une analyse sommaire, physico-chimique, de ces milieux. 


\section{BIOTOPE LARVAIRE}

Parmi les nombreux types d'étangs et de lagunes qui jalonnent les côtes du Golfe du Lion, celui de «La Figueirasse » correspond assez précisément à la «souillère » de G. Kuhnholtz-Lordat (1923). Ce terme couramment utilisé par les paysans et les manadiers de Petite-Camargue (inclus Bas-Gard) s'applique à des cuvettes sableuses de quelques hectares, noyées la majeure partie de l'année. La «souillère » communique

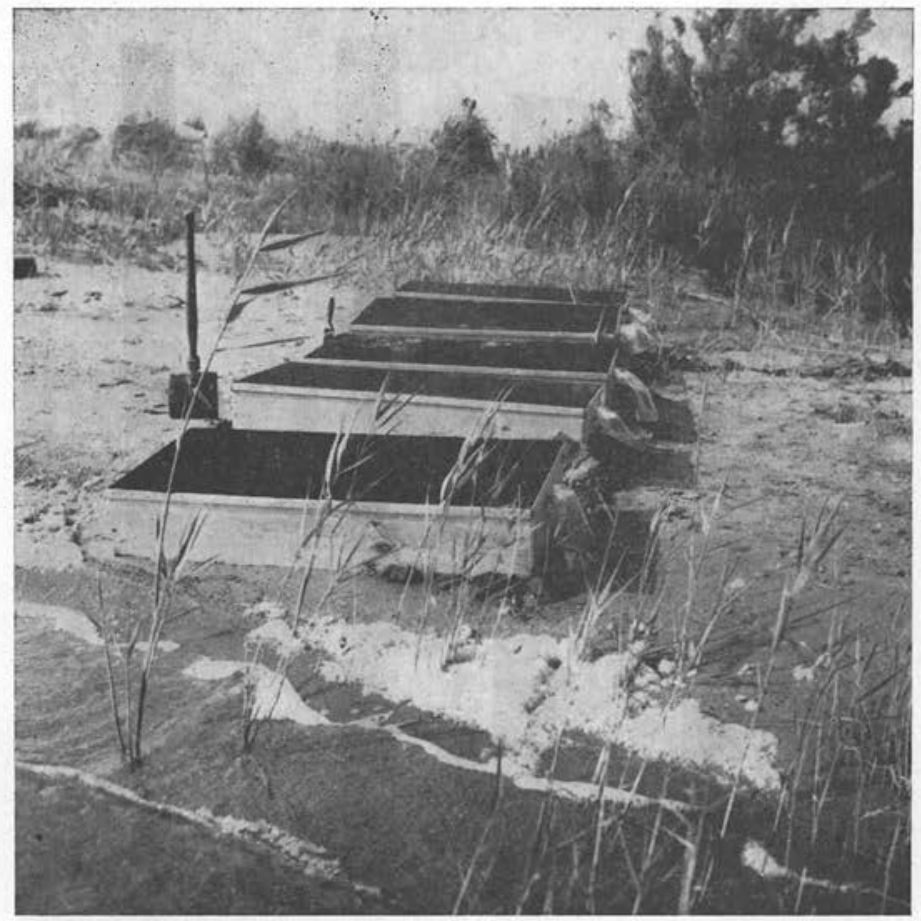

Fig. 5. - Biotope de Leptoconops (H.) kerteszi : plage sableuse en bordure de lagune à "La Figueirasse », 19 mai 1964. Au premier plan, Phragmites communis Trin.; à l'arrière-plan, frange de Juncus acutus L. précédant la dune à Amnophila arenaria (L.) Link et à Crucianella maritima L.; à droite boqueteau de Tamarix gallica $\mathrm{L}$.

avec la mer par des graus intermittents qui fonctionnent surtout à l'occasion des tempêtes d'équinoxe. L'eau de mer reste alors prisonnière jusqu'à son évaporation partielle ou totale vers la fin de l'été.

En bordure de la «souillère » s'étend une plage pratiquement dépourvue de végétation si ce n'est de rares touffes éparses de Salicornia radicans Sm. et Arthrocnemum 
glaucum Ung. Stern. Par place émergent quelques dunes en croissant couvertes de leur végétation classique à Oyat (Ammophila arenaria Link) ou Crucianelle (Crucianella maritima L.).

En raison du substratum sableux, la «souillère » est très sensible aux variations hydriques. Avec le beau temps se produit en effet un rapide recul des eaux, interrompu par quelques fluctuations dues à l'action combinée des vents et des orages. La lagune finit cependant par s'assécher par évaporation jusqu'à devenir une surface parfaitement exondée où l'on circule librement sans crainte d'enlisement. $\mathrm{A}$ la fin de l'été, les remontées de sels (« salants $»)$ forment par place de larges auréoles scintillantes. Rarement en raison de la nature sableuse du sol se produisent ici les fentes de retrait caractéristiques des «sols sonnants » de la Camargue ou du Languedoc.

Au sein de ce complexe écologique, le biotope larvaire de Leptoconops (H.) kerteszi est assez strictement localisé aux plages de sables récemment atterries. Au fur et à mesure du retrait des eaux, des étendues nouvelles sont libérées qui sont de nouveaux gîtes d'éclosion.

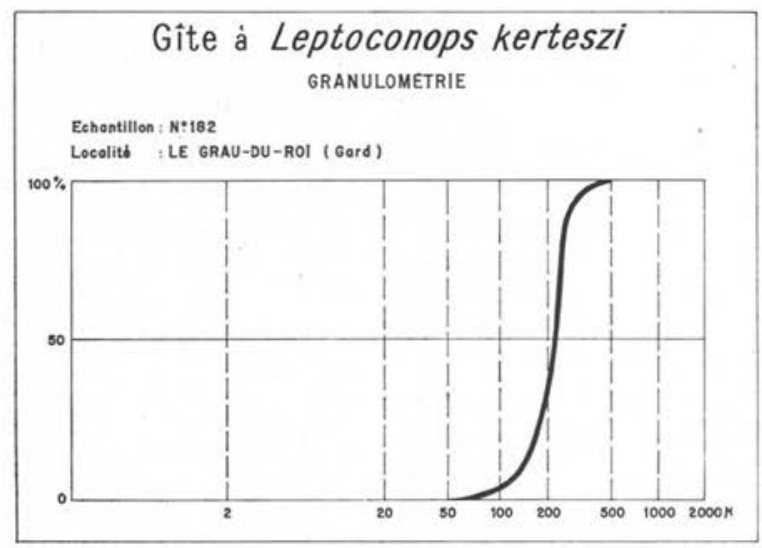

FIG. 6

La distance du gîte à l'eau libre de la lagune ne semble jouer que dans la mesure où se trouve modifiée corrélativement la hauteur de la nappe phréatique et par conséquent celle de la frange capillaire. C'est dire que les grèves inclinées ne * fonctionnent » que sur une auréole assez réduite alors que les surfaces très plates, parfois humides sur plusieurs centaines de mètres, donnent des gîtes extrêmement étendus. Lorsque la nappe descend au-dessous de $40 \mathrm{~cm}$, le gîte ne paraît plus « fonctionner ». Il peut toutefois redevenir actif à l'occasion d'un nouveau remplissage de la lagune (précipitations ou vents).

Qu'ils soient prélevés en surface, à 30,50 ou $80 \mathrm{~cm}$ de profondeur, les sables présentent le même caractère physique. Il s'agit de formations d'origine marine, enrichies 
d'une légère participation éolienne locale. Leur analyse granulométrique les classe dans la fraction 177-200 $\mu$ (fig. 6). Ils contiennent environ $95 \%$ de quartz et quelques métaux lourds.

TABLeAU I. - Biotope de Leptoconops (H.) kerteszi Kieffer, 1908

ANALYSE GRANULOMÉTRIQUE DES SOLS

\begin{tabular}{|c|c|c|c|c|c|}
\hline \multirow{2}{*}{ Texture } & \multirow{2}{*}{$\begin{array}{c}\text { DIAMÈTRE DES } \\
\text { PARTICULES } \\
\text { (en } \mu)\end{array}$} & \multicolumn{4}{|c|}{$\begin{array}{c}\text { PoIDS } \\
\text { (pour } 100 \text { grammes) }\end{array}$} \\
\hline & & $\mathrm{n}^{\circ} \quad 1$ & $\mathrm{n}^{\circ} 2$ & $\mathrm{n}^{\circ} 3$ & $\mathrm{n}^{\circ} 4$ \\
\hline \multirow{3}{*}{$\begin{array}{l}\text { Sables } \\
\text { grossiers }\end{array}$} & 2.000 & & & & \\
\hline & 707 & 0,02 & 0,05 & 0,07 & 0,02 \\
\hline & & 0,2 & 0,60 & 0,4 & 0,3 \\
\hline \multirow{2}{*}{$\begin{array}{l}\text { Sables } \\
\text { moyens }\end{array}$} & 254 & 0,6 & 1,9 & 0,7 & 0,8 \\
\hline & & 25,6 & 19,4 & 19,5 & 14,9 \\
\hline \multirow{3}{*}{$\begin{array}{l}\text { Sables } \\
\text { fins }\end{array}$} & & 62,2 & 59,7 & 62,8 & 65,2 \\
\hline & & 7.8 & 12,1 & 10,9 & 11,9 \\
\hline & & 3,5 & 4,9 & 4,9 & 5,9 \\
\hline $\begin{array}{l}\text { Limons } \\
\text { s.1. }\end{array}$ & & 0,05 & 1,4 & 0,8 & 1,0 \\
\hline
\end{tabular}

L'analyse physico-chimique des échantillons d'eau prélevée dans la nappe phréatique à la hauteur des gîtes à Leptoconops montre une assez grande homogénéité dans la concentration ionique.

Dans l'ensemble, leur minéralisation est en effet importante (cf. tableau II) à l'encontre de celle des eaux prélevées sous les dunes libres ou fixées, c'est-à-dire dans des biotopes non colonisés par les larves de Leptoconops. Précisons que cette différence de concentration entre les nappes phréatiques dunaires et lagunaires est classique, la dune comportant toujours un «noyau» central d'eau douce provenant des précipitations directes. 


\section{REPARTITION GEOGRAPHIQUE}

Leptoconops (Holoconops) kerteszi Kieffer, 1908, avec ses variétés peneti Langeron, 1913, americanus Carter, 1921 et son synonyme $L$. (H.) nachistchevanicus Dzhafarow, 1961, possède une aire de répartition relativement vaste (2). Il est en effet connu

\section{Biotope de Leptoconops (H.) kerteszi Kieffer, 1908}

ANALYSE PHYSICO-CHIMIQUE DE LA NAPPE PHRÉATIQUE

\begin{tabular}{|c|c|c|c|c|c|c|}
\hline Prélèvement $\mathrm{n}^{\circ}$ & (1) & (2) & (3) & (4) & (5) & (6) \\
\hline $\begin{array}{l}\text { Hauteur de la nappe } \\
(\mathrm{en} \mathrm{cm}) \quad \ldots \ldots \ldots \ldots\end{array}$ & 11 & 13 & 18 & 25 & 35 & 80 \\
\hline Chlore $(g / 1) \quad \ldots$. & 8,4 & 6,90 & 7,40 & 6,20 & 4,50 & 0,140 \\
\hline Sodium $(g / 1) \quad \ldots$. & 4,54 & 4,23 & 3,68 & 3,19 & 2,90 & 0,130 \\
\hline Potassium $(\mathrm{g} / \mathrm{l}) \ldots$ & 0,225 & 0,200 & 0,195 & 0,157 & 0,130 & 20 \\
\hline Calcium $(\mathrm{g} / \mathrm{l}) \ldots$ & 0,120 & 0,160 & 0,110 & 0,130 & 0,160 & 70 \\
\hline Magnésium $(g / \mathrm{l}) \ldots$ & 0,050 & 0,018 & 0,048 & 0,035 & 0,007 & 6 \\
\hline Résistivité (ohm-cm) & 36 & 46 & 38 & 43 & 70 & 1276 \\
\hline Température $\left({ }^{\circ} \mathrm{C}\right)$ & 23 & 20 & 22 & 20 & 17 & 18 \\
\hline$p \mathrm{H} \quad \ldots \ldots \ldots \ldots$ & 6,70 & 7,85 & 6,65 & 6,75 & 7,70 & 7,85 \\
\hline
\end{tabular}

d'Afrique (Egypte, J. Kieffer, 1908; Tunisie, M. Langeron, 1913 ; Nord-Tchad, J. Clastrier, J.-A. Rioux et S. Descous, 1961) ; d'Asie (Palestine, E.-E. Austen, 1921 ; Azerbeijan, Arménie, S. Dzhafarow, 1961; d'Europe ( Midi » méditerranéen, H. Harant et D. Brunel, 1950) et d'Amérique (Utah, H. Carter, 1921 ; Californie, S. B. Freeborn et E. C. Zimmermann, 1934 ; Wyoming, Nebraska, Wirth, 1951 ; Montana, Colorado, Nouveau-Mexique, W. W. Wirth, 1952 ; Colombie Britannique, Curtiss, 1957).

\section{Résumé}

Après un bref rappel morphologique du Cératopogonide Leptoconops (Holoconops) kerteszi Kieffer, 1908, les auteurs décrivent une méthode de piégeage permettant la prospection rationnelle des zones marécageuses du «Midi » méditerranéen. Le gîte larvaire de l'espèce est découvert dans une région sableuse du Bas-Gard (domaine de La Figueirasse). Il s'agit de grèves sableuses en bordure de lagune. Le mâle est capturé pour la première fois en France. Les caractéristiques physionomiques, physiques et chimiques du biotope sont rapidement analysées.

(Laboratoire d'Ecologie médicale et de Pathologie parasitaire de la Faculté de médecine Montpellier [Hérault, France]

(2) Remarquons toutefois que la plupart de ces territoires sont soumis au régime pluviothermique méditerranéen ou sub-méditerranéen.

Annales de Parasitologie humaine et comparée (Paris), t. 40, 1965, $\mathrm{n}^{\circ} 2$ 


\section{Bibliographie}

Austen (E. E.), 1921. - A Contribution to Knowledge of the Blood-Sucking Diptera of Palestine other than Tabanidae. Bull. Ent. Res., 12, p. 107.

Boesel (M. W.), 1948. - Holoconops in the Western Lake Erie Region (Diptera : Heleidae). The Ohio Journal of Science, 48, 69-72.

Callot (J.) et Kremer (M.), 1961. - Culicoides riouxi et Culicoides pseudo-heliophilus, espèces nouvelles du groupe des Culicoides à ailes sans taches (Diptera ; Ceratopogonidae). Ann. Parasitol., 36, 677-688.

CARTer (H. F.), 1921. - A revision of the genus Leptoconops Skuse. Bull. Ent. Res., 12, 1-28.

Carter (H. F.), Ingram (A.) et MacFie (J. W. S.), 1920. - Observations on the Ceratopogonine midges of the Gold Coast with descriptions of new species. I-II. Ann. Trop. Med. Parasit., 14, 187-274.

Clastrier (J.), Rioux (J. A.) et Descous (S.), 1961. - Notes sur les Cératopogonidés. XII. Cératopogonidés du Nord-Tchad. Arch. Inst. Past. Alg., 39, 49-98.

Dzhafaroy (S.-M.), 1961. - Description de trois espèces hématophages (Heleidae, Diptera) du Petit Caucase. Izv. Akad. Nauk. Azerbajdzanskoj S.S.R. 10, 75-80.

EMBerger (L.), 1942. - Un projet de classification des climats du point de vue phytogéographique. Bull. Soc. Hist. Nat., Toulouse, 77, 97-124.

ENDERLEIN (G.), 1936. - Zweifluger. Die Tierwelt Mitteleuropas, 6, 49-50.

Foley (H.) et Picout-Laforest (A.), 1923. - Quelques observations sur la répartition et la biologie d'Holoconops mediterraneus dans les régions sahariennes. Arch. Inst. Past. Alg. 1, 684-687.

Fontaine (R. E.), Green (D. H.) et Smith (L. M.), 1957. - Ecological observations of the Valley Black Gnat, Leptoconops torrens Townsend. J. Econ. Entomol., 50, 764-767.

Freeborn (S. B.) et Zimmerman (E. C.), 1934. - The males of Leptoconops torrens Tns., and L. kerteszi Kieff. Bull. Ent. Res., 25, 257-262.

Grant (C. D.) et LaURET (T. H.), 1957. - Initial study on Leptoconops torrens (Townsend) by the San Mateo County Mosquito Abatement District. California Vector Views, 4, 29-32.

Hannoun (C.), Beytout (D.), Joubert (L.), Mouchet (J.) et Abonnenc (E.), 1964. - Rapport général sur la deuxième Mission d'Etude des Arbovirus en Camargue et en Bas-Languedoc, 15 juin-5 juillet (rap. ronéo).

Harant (H.), 1937. - Les «arabis» moucherons vulnérants. Arch. Soc. Sc. Med. Montp., p. 265.

Harant (H.) et Brunel (D.), 1950. - Sur les Leptoconops s. lato (Dipt. Heleidae) du Midi méditerranéen. Vie et Milieu, 240-242.

Harant (H.) et Galan (G.), 1943. - Notes sur les Diptères de la Région méditerranéenne. VII. Remarques sur les Leptoconops: Leptoconops lisbonnei n. sp. Bull. Soc. Path. Exot., 36, 257. 
Huttel (W.) et Huttel (N.), 1951. - A propos de Leptoconops inopinatus n. sp. de la région de Montpellier. Bull. Soc. Ent. Fr., 45-47.

Kieffer (J. J.), 1908. - Description d'une espèce nouvelle de Chironomide d'Egypte. Ann. Mus. Nat. Hungar., 6, 576-577.

KiefFer (J.-J.), 1918. - Chironomides d'Afrique et d'Asie conservés au Muséum National Hongrois de Budapest. Ann. Mus. Nat. Hist. Hungar., 16, 31-136.

KuhnholtZ-LoRdat (G.), 1923. - Essai de Géographie botanique sur les dunes du Golfe du Lion. Thèse Sciences, imp. La Presse, Montpellier, 34 p.

Langeron (M.), 1913. - Mycterotypus laurae n. sp. Arch. de Parasitol., 16, 282.

LASSERre (O.), 1947. - Contribution à l'étude des moucherons piqueurs Cératopogonides. Thèse Médecine, Montpellier.

MACFIE (J. W. S.), 1943. - Ceratopogonidae (Diptera) from Egypt. Proc. R. Ent. Soc. London (B), 12, 145-159.

Mulla (S.), 1958. - Unusual occurence of L. kerteszi. California Vector Views, 5, 53-56.

Roman (E.), 1937. - Sur quelques Arthropodes vulnérants récoltés en Camargue. Bull. Soc. Ent. Fr., 131-133.

SkuSE (A. A.), 1890. - Diptera of Australia. Part. VI. The Chironomidae. Proc. Linn. Soc N.S. W., 4, 215-313.

Smith (L. M.) et Lowe (H.), 1948. - The Black Gnats of California. Hilgardia, 18, 157-183.

Whitsel (R. H.) et Vickery (C. A. J.), 1962. - Progress report on the Black Gnat, Leptoconops torrens Townsend, research project.

WIRTH (W. W.), 1951. - A new biting midje of the genus Leptoconops from Florida, with new records of other american species. Proc. Ent. Soc. Wash., 53, 281-284.

Wirth (W. W.), 1952. - The Heleidae of California. Univ. Calif. Publ. Ent., 9, 95-266. 